\title{
BMJ Open Persistent opioid use and opioid-related harm after hospital admissions for surgery and trauma in New Zealand: a population-based cohort study
}

\author{
Jiayi Gong (D) , ${ }^{1,2}$ Alan Forbes Merry (D) , ${ }^{3,4}$ Kebede A Beyene, ${ }^{1}$ Doug Campbell, ${ }^{3,4}$ \\ Chris Frampton, ${ }^{5}$ Peter Jones, ${ }^{6}$ John McCall, ${ }^{6}$ Matthew Moore, ${ }^{3}$ \\ Amy Hai Yan Chan (D) ${ }^{1,2}$
}

To cite: Gong J, Merry AF, Beyene KA, et al. Persistent opioid use and opioid-related harm after hospital admissions for surgery and trauma in New Zealand: a populationbased cohort study. BMJ Open 2021;11:e044493. doi:10.1136/ bmjopen-2020-044493

- Prepublication history and additional material for this paper is available online. To view these files, please visit the journal online (http://dx.doi.org/10. 1136/bmjopen-2020-044493).

Received 04 September 2020 Revised 24 December 2020 Accepted 30 December 2020

Check for updates

(C) Author(s) (or their employer(s)) 2021. Re-use permitted under CC BY-NC. No commercial re-use. See rights and permissions. Published by BMJ.

For numbered affiliations see end of article.

Correspondence to

Dr Amy Hai Yan Chan;

a.chan@auckland.ac.nz

\section{ABSTRACT}

Introduction Opioid use has increased globally for the management of chronic non-cancer-related pain. There are concerns regarding the misuse of opioids leading to persistent opioid use and subsequent hospitalisation and deaths in developed countries. Hospital admissions related to surgery or trauma have been identified as contributing to the increasing opioid use internationally. There are minimal data on persistent opioid use and opioid-related harm in New Zealand (NZ), and how hospital admission for surgery or trauma contributes to this. We aim to describe rates and identify predictors of persistent opioid use among opioid-naïve individuals following hospital discharge for surgery or trauma.

Methods and analysis This is a population-based, retrospective cohort study using linked data from national health administrative databases for opioid-naive patients who have had surgery or trauma in NZ between January 2006 and December 2019. Linked data will be used to identify variables of interest including all types of hospital surgeries in NZ, all trauma hospital admissions, opioid dispensing, comorbidities and sociodemographic variables. The primary outcome of this study will be the prevalence of persistent opioid use. Secondary outcomes will include mortality, opioid-related harms and hospitalisation. We will compare the secondary outcomes between persistent and non-persistent opioid user groups. To compute rates, we will divide the total number of outcome events by total follow-up time. Multivariable logistic regression will be used to identify predictors of persistent opioid use. Multivariable Cox regression models will be used to estimate the risk of opioid-related harms and hospitalisation as well as allcause mortality among the study cohort in a year following hospital discharge for surgery or trauma.

Ethics and dissemination This study has been approved by the Auckland Health Research Ethics Committee (AHREC- AH1159). Results will be reported in accordance with the Reporting of studies Conducted using Observational Routinely collected health data statement (RECORD).

\section{BACKGROUND}

Opioids are widely prescribed in hospitals for acute, chronic non-cancer-related and

\section{Strengths and limitations of this study}

- This will be the first study in New Zealand to examine the relationship between hospital admission for surgery or trauma and the incidence of persistent opioid use.

- The study will include 14 years of opioid usage data, making this one of the largest opioid usage studies to date (2006-2019).

- A limitation of the study lies in its use of retrospective secondary data, which has limited control over confounders.

cancer-related pain. However, in the last decade, opioid addiction and dependency have contributed substantially towards hospitalisation and death globally, especially in the USA. ${ }^{1}$ Mortality related to opioid overdose accounts for nearly 530 deaths per week in the USA. ${ }^{1}$ In New Zealand (NZ), a study in 2017 showed from 2008 to 2012, a total of 325 deaths were primarily ascribed to opioid use. ${ }^{2}$ Of these, 179 deaths were from unintentional overdose and may be preventable. ${ }^{2}$ No studies have examined recent trends in NZ. In NZ, five strong opioids and a total of 33 different formulations are licensed for prescribing in pain management: 14 for morphine, 9 for oxycodone, 5 for fentanyl, 4 for methadone and 1 for pethidine. Three weak opioids and a total of 9 different formulations are available for prescribing: 5 for tramadol, 3 for codeine and 1 for dihydrocodeine.

The NZ Health Quality \& Safety Commission (HQSC) in $2016^{3}$ reports that opioid use has increased in NZ with 16.6 per 1000 people compared with 14.4 per 1000 in 2011. The rate of persistent opioid use, defined by the HQSC as use of 6 weeks or longer, has not changed between 2011 and 2016 and is around 2 per 1000 patients. The report 
also showed that almost half of the patients dispensed a strong opioid had a hospital admission in the week prior to opioid prescribing in the community.

Internationally, persistent opioid use is generally defined as opioid use for 90 days or longer. ${ }^{4}$ Studies from the USA and Canada have indicated the rate of persistent opioid use beyond 90 days to be between $0.1 \%$ and $8.2 \%$ in the context of pain management after surgery and trauma. ${ }^{5}$ In the acute setting, higher rates of opioid prescribing by emergency physicians has also been linked to subsequent long-term opioid use in the USA (OR $1.30,95 \%$ CI 1.23, 1.37, $\mathrm{p}<0.001){ }^{6}$

Hospital admissions may act as drivers for subsequent persistent opioid use in the community setting. NZ data show that younger people (under 24 years of age) were more likely to have a hospital admission prior to the dispensing of a strong opioid than older people (80 years and over)-rates opioid dispensing were 7 per 10 people in younger people versus 3.7 per 10 in those above $80 .^{3}$ This usage rate may be due to clinicians' perception that opioids are generally safer in younger patients because they are more likely to have normal metabolic functions. However, studies have suggested that addictive behaviour tends to be over-represented in younger patients (18 -29 years old),${ }^{78}$ mainly concerning other substances of abuse such as cannabis and cocaine. Our study will examine persistent opioid use and opioid-related harm across all age groups, to describe how these rates may change across ages.

Outcomes related to persistent opioid use have also been poorly evaluated both in NZ and elsewhere. A recent study published by a Swedish group showed that post-trauma patients with persistent opioid use carry an excess risk of mortality, even after adjustment for age, sex, somatic comorbidity, psychiatric-morbidity, substance abuse, injury severity and admissions to intensive care (HR 1.82, 95\% CI 1.34 to $2.48, \mathrm{p}<0.001) .{ }^{9}$ There are few mortality data in NZ related to persistent opioid use, despite the presence of national databases recording health outcomes.

In NZ, the opportunity for population-based cohort studies is unique in several ways. First, NZ has a single universal healthcare system; the government covers $80 \%$ of the cost of healthcare. All NZ citizens, permanent residents and certain work visa holders have access to free or low-cost physician services, hospital care and prescription medicines. ${ }^{10}$ Physician services are private businesses that set their consultation fee, but the fees are set by the government and cannot exceed a certain limit. Patients enrolled in a specific physician service will have a lower cost per visit, as the government would partially fund the visit. ${ }^{10}$ Most physician services are free for patients 13 years or under. Most prescription medicines are fully funded by the government, with the patient having to copay only a dispensing fee of NZ $\$ 5$ per item to the pharmacy. ${ }^{11}$ Second, all patients accessing the health and disability system will be assigned a unique identifier code, in both private and public funded hospitals, called the National
Health Index (NHI) number. ${ }^{12}$ The Ministry of Health $(\mathrm{MOH})$ uses the NHI number in an encrypted form so that data can be used for statistical purposes to report on the health of the NZ population. ${ }^{12}$ This then allows for linkage of data across different datasets for research, for example, hospital admissions, medication dispensing and mortality can be linked. Third, it is possible to track all admissions to public and private hospitals. This permits the calculation of an emerging measure called days alive and out of hospital (DAOH). DAOH is a composite outcome that integrates several clinically significant outcomes, including mortality, hospital length of stay and hospital readmission. ${ }^{13}$ It is sensitive to any complication of surgery that prolongs an admission, leads to a readmission or results in death. Fourth, data on ethnicity are routinely collected by the $\mathrm{MOH}$. NZ has a large indigenous population (Māori) who experience substantial disadvantages in health status and outcomes compared with the non-Māori population. ${ }^{14}$ A recent study assessing the use of non-opioid analgesia between ethnicities in NZ showed a significant disparity in outcome between Māori and non-Māori patients, with Māori patients having more in adverse events, with a relative risk 2.54 (95\% CI 2.23 to 2.90). ${ }^{15}$ The likely risk of persistent opioid use and subsequent opioid-related harm in NZ may also be distributed unequally across ethnic groups. The NZ healthcare system is obligated to address these inequities under the protection principle of the Treaty of Waitangi. ${ }^{16}$

Thus, we aim to determine the patterns of opioid use and opioid-related harm in NZ following discharge from hospital for surgery or trauma over 14 years from 1 January 2006 to 31 December 2019. Specifically, in this study, we aim to determine the rates of persistent opioid use post-discharge from the hospital and evaluate outcomes including opioid dependence, opioid-related overdose, opioid-related hospitalisation and mortality. We will also measure $\mathrm{DAOH}$ at 365 days $\left(\mathrm{DAOH}_{365}\right)$, as an outcome that will be used for measuring the quality of perioperative care and postoperative recovery. ${ }^{13}$ We will also identify the predictors of these outcomes.

\section{METHODS AND ANALYSIS}

\section{Study design and setting}

This will be a population-based retrospective cohort study involving residents of NZ using linked, routinely collected health data. We plan to start data collection and analysis by 1 March 2021 and complete the study within 12 months.

\section{Sources of data}

The study will use several national administrative health databases, which can be linked via an encrypted, unique patient identifier known as the NHI. ${ }^{12}$ To examine hospitalisations data, we will use the National Minimum Data Set (NMDS). ${ }^{17}$ The NMDS is a national registry of all inpatient admissions reported to the $\mathrm{MOH}$. The NMDS includes information on dates of hospital stay, 
diagnoses and medical procedures. All medical procedures are coded according to the Australasian Classification of Health Interventions (ACHI). ${ }^{18}$ In NMDS, primary and secondary diagnoses are coded according to the International classification of diseases 10th editionAustralian modified version (ICD-10AM). The NMDS captures $99 \%$ of all operations performed in NZ. ${ }^{19}$ The National Non-Admitted Patients Collection (NNPAC) is the national database used to capture information on patients who presented to the emergency department and spent less than 3 hours in a hospital. This database will be linked with data from the Accident Compensation Corporation (ACC) to identify patients admitted to an NZ hospital with trauma but did not require prolonged hospital admission. ${ }^{20}$ ACC is NZ's Crown entity responsible for administering the country's no-fault accidental injury compensation scheme. ${ }^{21}$ The Mortality Collection (MORT) is a national database that classifies the underlying cause of death for all deaths registered in NZ. MORT uses the ICD-10AM classification for mortality coding. ${ }^{22}$ Mortality-related outcomes will be retrieved from this database.

Data on opioid dispensing and other medicines prescribed to patients receiving opioids will be obtained from the Pharmaceutical Collection (Pharms). Pharms is a data warehouse of information related to pharmaceutical subsidies and contains claim and payment information from pharmacists for subsidised dispensing. ${ }^{23}$ The Pharms database contains information on all prescriptions dispensed by community pharmacies in NZ since 1998, according to local codes akin to the Anatomical Therapeutic Chemical classification system codes. The database includes medication name, quantity, days of supply, formulation, date of refill, route of administration and codes identifying the therapeutic category of the medication. Medicines dispensed in hospitals are not included in the database. Patients meeting the inclusion criteria will be determined from the NMDS and NNPAC plus ACC then matched with their respective opioid dispensing data from Pharms via their respective encrypted NHI.

Other databases that we will access include the NHI and Primary Health Organisation enrolment. These datasets will be used to retrieve information relevant to the patient, including demographic, gender, age, ethnicity and socialeconomic status. ${ }^{24}$

\section{Study cohort}

The study design is illustrated in figure $1 .^{25}$ The study population includes all opioid-naive patients (of any age) who had a surgical procedure or presented to the hospital with trauma in one of NZ's 39 public hospitals between 1 January 2006 and 31 December 2019. Surgical patients will be defined as all individuals in the NMDS assigned a surgical procedure and associated anaesthetic code via the ACHI. Trauma patients with a hospital stay of greater than 3 hours will be identified using ICD-10AM codes for trauma in the NMDS dataset. Individuals with less than 3 hours of admission will only be registered on
NNPAC. Thus their data will likely require linkage of ACC database via ACC injury codes and claimant number to obtain event details.

The date of hospital discharge following the initial surgical or traumatic event and exposed to opioids will be considered as the index date. For patients with more than one admission for surgical procedures or trauma during the study period, only the first admission will be included in the study cohort. Individuals will be considered opioid-naïve if they do not have dispensing records for any opioid medications in 180 days before the index date. We will exclude the following types of patients: (1) those who have evidence of a surgical procedure or traumatic event in 365 days prior to the index date and (2) those with evidence of opioid use disorders (eg, overdose, misuse or dependence) or opioid prescriptions in 180 days prior to the index date. Patients who underwent multiple procedures in 24 hours will be examined separately as their likely risk of persistent opioid use may be different from other patients.

We will follow up all patients who meet the study inclusion criteria for a maximum of 365 days, or until subsequent hospital admission for surgery or trauma, the last opioid dispensing claims date, occurrence of the outcomes of interest, or death from any cause, whichever comes first—see figure 1 for study design.

Postoperative prescribing of opioids is generally guided by the Australian and New Zealand College of Anaesthetists guidelines. ${ }^{26}$ Multimodal analgesia is used instead of mainly opioid-based analgesia to improve pain control and reduce opioid consumption and adverse effects. ${ }^{26}$

See online supplemental material 1 for surgical procedures and ICD-10AM codes for trauma-related admissions

\section{Outcome assessment}

The primary outcome will be persistent use of opioids after trauma or surgery. As in previous studies of opioid use, ${ }^{27}{ }^{28}$ persistent opioid use will be defined as continuous pharmacy claim for one or more opioid prescription 91 days to 365 days after the index date. Patients who continuously filled one or more opioid prescriptions within 90 days after the index date but did not thereafter will be considered as non-persistent opioid users. Characteristics of persistent and non-persistent opioid users will be compared with identify predictors of persistent opioid use. Our secondary outcomes will include total duration of persistent opioid use from the index date, opioid-related fatal overdose, all-cause mortality, opioid dependency, opioid misuse and hospitalisation related to opioid overdose and toxicity during the follow-up period. We will also measure $\mathrm{DAOH}_{365}$.

Mortality due to opioid overdose will be defined as the presence of a date of death and opioid overdose as the cause of death as recorded in the MORT database via ICD-10AM codes. These episodes of related harm will be identified during patients' subsequent admission after the index date. 


\section{Cohort Entry Date (Index date) \\ (First surgery or trauma event) \\ Day 0}

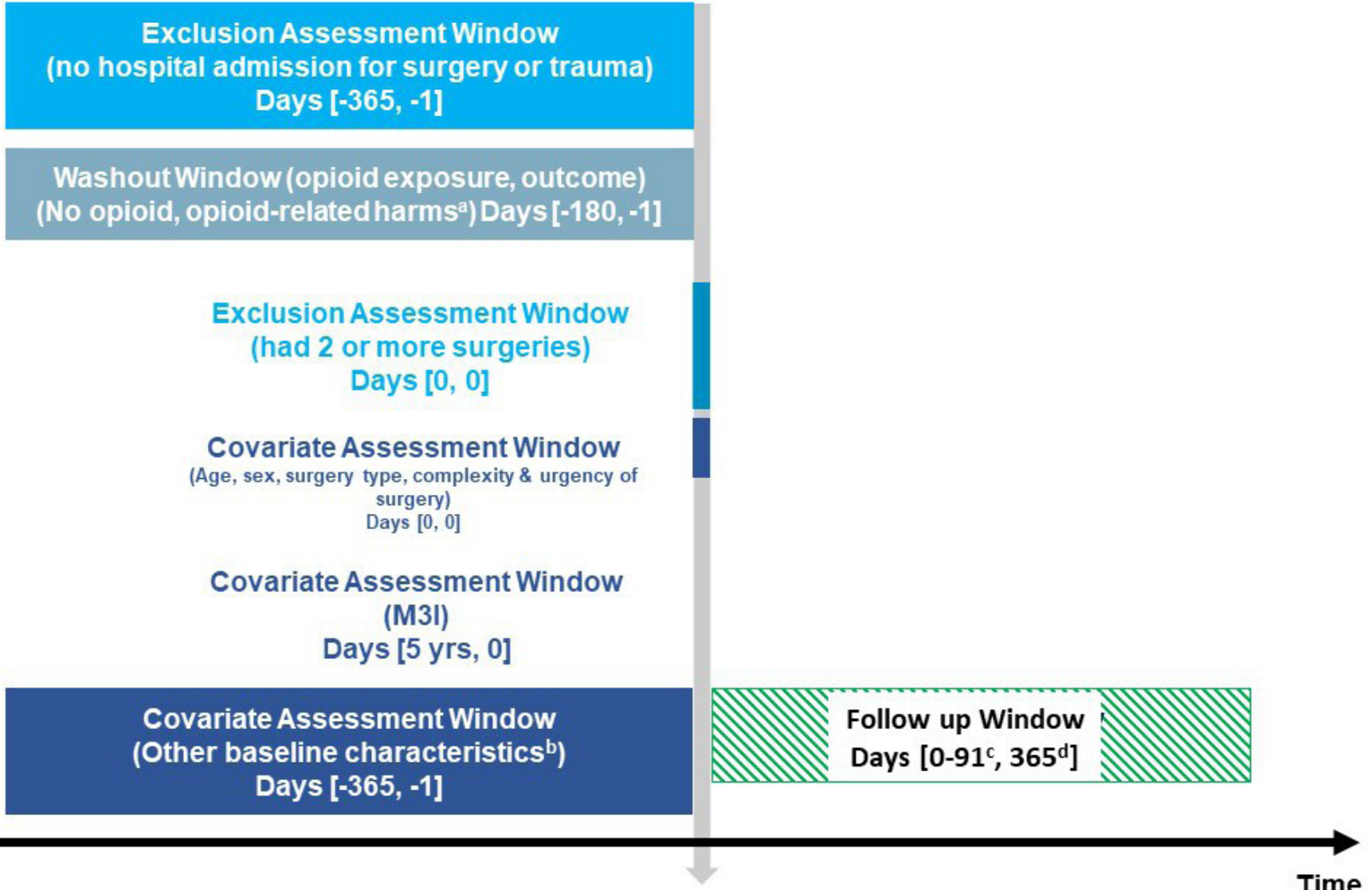

a. Opioid-related harms include opioid use disorders (e.g. overdose, misuse or dependence)

b. Other baseline characteristics include: depression (ICD 104M codes: F32 -F33), anxiety (F40-F48), dementia (FOO-FO3),

schizophrenia and related psychotic conditions (F20-F29), bipolar disorder (F31), alcohol and nicotine dependence (F10.20-

$\mathrm{F} 10.29, \mathrm{~F} 17.200$ - F17.299), substance use (F10-F16, F18-F19, F55), mood disorder (F30-F39), other mental health conditions

(F04-F09, F51-F53, F59, F63, F68, F69, F930-F932, F99), other medications (paracetamol, NSAIDs, pregabalin, gabapentin,

benzodiazepines, antipsychotics, antidepressants) and presence of chronic pain

c. Earliest outcome of interest. persistent opioid beyond 90 days

d. Secondary outcome at 365 days: total duration of persistent opioid use from an index date, opioid-related fatal overdose, all-

cause mortality, opioid dependency, opioid misuse, and hospital admission related to opioid overdose and toxicityduring the

follow up period.

M31 = M3 Comorbidity Index

Figure 1 Study design diagram with cohort entry criteria including windows of time for exclusion assessment, opioid washout, covariate assessment, baseline characteristics assessment and follow-up period. ICD-10AM, International classification of diseases 10 th edition-Australian modified version.

See the online supplemental material 1 for secondary outcome-related ICD-10AM.

\section{Opioid exposure assessment}

The primary exposure in this study is the receipt of a new opioid prescription after a surgical or traumatic event. All patients who filled at least one opioid prescription within 7 days of discharge will be considered exposed to prescription opioids and will be included in the study cohort. The 7-day grace period assumes that an opioid prescription filled by an opioid-naïve individual during this period is likely to be used to treat acute pain after surgery or trauma, and any opioid use after 7 days is unlikely to be related to the surgery or trauma event. Opioid use will be assessed by date of dispensing, dose, frequency, days or quantity supplied, and frequency (where available) of dispensing to infer the duration of use.

Of the different opioid formulations available in NZ, only topical (fentanyl only) and oral preparations will be included. Patients discharged with parenteral products will be excluded. The indication for parenteral opioids is unlikely related to postoperative pain management but more likely for other indications such as palliation.

To account for patients being on different strong opioids with variable potency, patients daily opioid dose will be standardised by converting the prescribed dose to oral morphine equivalence (OME) in milligrams to account for overall opioid exposures relative to morphine, 
per formula below-see online supplemental material 1 for OME conversion factors: ${ }^{29} 30$

OME $=$ PackStrength $\times$ OMEconversionfactor $\times$ Quantitydispensed

That is, if 30 capsules of oxycodone $5 \mathrm{mg}$ were dispensed, this would equate to an OME of $225 \mathrm{mg}$.

Cessation of opioid use will be defined as a pause of medication supply for more than 45 days from the last dispensing. This is because the maximum legal period of supply for opioids in NZ is 30 days. To account for any leftover of supply a further 15 days will be included.

\section{Covariates of persistent opioid use and opioid-related harm}

We will aim to identify predictors of persistent opioid use and other secondary outcomes of interest, including opioid-related mortality. Various predictors have been investigated previously, including age, type of surgery, length of stay for the index surgery or trauma event, surgical specialty (cardiac, vascular, digestive, thoracic, neurosurgery, musculoskeletal, urinary and other), multiple surgeries in 1 hour, complexity and urgency of surgery. ${ }^{31}{ }^{32}$ These will be collected via the NMDS in addition to ethnicity, gender, socioeconomic status, social deprivation score (via NZ Deprivation Index-NZDep). ${ }^{33}$ The NZDep is an area-based measure of socioeconomic deprivation in NZ. The urgency of the surgery will be categorised as elective or acute. The operative complexity will be categorised using the Johns Hopkins five-category classification, where each procedure will be given an operation severity grade from one to five. ${ }^{34}$ The method of identifying all procedures performed in NZ hospitals is consistent with a previously published paper involving current coinvestigators on postoperative mortality risk. ${ }^{19}$

Other comorbidities will be collected that have been shown in the literature to be predictors of persistent opioid use ${ }^{531} 32$ such as depression (ICD-10AM codes: F32F33), anxiety (F40-F48), dementia (F00-F03), schizophrenia and related psychotic conditions (F20-F29), bipolar disorder (F31), alcohol and nicotine dependence (F10.20-F10.29, F17.200-F17.299), substance use (F10F16, F18-F19, F55), mood disorder (F30-F39), other mental health conditions (F04-F09, F51-F53, F59, F63, F68, F69, F930-F932, F99) and presence of chronic pain in a year prior to index date. For each patient included in the cohort, the M3 index (M3I) score and the American Society of Anesthesiologists (ASA) physical status classification system ${ }^{35}$ will be used as a measure of comorbidity using information from any inpatient or outpatient contact the 5 years before the index date ${ }^{36}$ M3I score will be computed from a list of 61 health conditions based on the presence/absence of previous diagnostic codes (see online supplemental material 1 for the specific health conditions). We will use the ASA score on each patients' NMDS record at the time of surgery. Additionally, we will adjust for the year of cohort entry in multivariable models assessing the relationship between explanatory variables and outcomes of interest.
To assess concurrent medications use, we will examine patient's medications at baseline and in 6 months prior to the index date using the Pharms database. Concomitant medications that are potential predictors for persistent opioid use include non-opioid analgesia, including paracetamol, pregabalin, gabapentin, benzodiazepines, antipsychotics, mood stabilisers and antidepressants. Additionally, information on non-steroidal anti-inflammatories dispensing will be collected as these have been shown to predict persistent opioid use. ${ }^{3037}$

\section{Data analysis}

Descriptive statistics will be used to describe baseline characteristics and the outcomes of interest. Continuous variables will be described using the mean and SD or median and IQR as appropriate, depending on data distribution. Categorical variables will be described using frequency distributions and percentages. Kolmogorov-Smirnov and Shapiro-Wilk tests of normality will be used to assess the distribution of continuous variables. Parametric and non-parametric bivariate tests (eg, Student's t-test, MannWhitney $\mathrm{U}$ test or $\chi 2$ test) will be used to compare the characteristics of individuals who may or may not exhibit persistent opioid use after surgery or trauma.

A multivariable logistic regression model will be used to examine the association between potential predictors and persistent opioid use after surgery/trauma. ORs with $95 \%$ CIs will be reported for each predictor. All predictor variables will be included simultaneously in the multivariable regression model. Multicollinearity between variables will be assessed using variance inflation factors and tolerance.

We will calculate the crude rate of each outcome of interest for both persistent and non-persistent opioid user groups. To compute the rates, we will divide the total number of outcome events by total follow-up time. These will be reported as cases per 1000 person-days with confidence intervals derived from a Poisson or negative binomial distribution as appropriate. Cox multivariate regression models will be used to estimate the risk of all-cause mortality, opioid-related harms and hospitalisation associated with persistent use of opioids in the 365 days after the index date for all patients. HRs and $95 \%$ CIs will be reported. The proportional hazards assumption of the Cox model will be tested using log-minus-log plots, and C-statistics will be used to identify the C-index of the multivariable Cox regression models. The KaplanMeier method will be used to summarise survival time for persistent and non-persistent opioid users. A two-sided $\mathrm{p}<0.05$ will be considered statistically significant for all statistical tests. We will make appropriate corrections to allow for multiple statistical tests. We will undertake all analyses using the $\mathrm{R}$ statisticals software package ( $\mathrm{R}$ V.4.0.2).

\section{Subgroup analysis}

We will repeat the primary analyses by stratifying across three age groups $(<18,18-44,45-64$ and $\geq 65$ years $)$ to examine whether the strength of the predictors for 
persistent opioid use and secondary outcomes vary across age. We will also assess whether the findings of the primary analysis are consistent across subgroups defined according to sex, ethnicity (Māori vs non- Māori), different opioids, urgency of surgery (elective vs non-elective) or surgery complexity via operation severity (grades 1-5).

\section{Sensitivity analysis}

To assess the robustness of our primary analyses, we will conduct several sensitivity analyses. As noted, our primary analysis will include all patients in the cohort, regardless of whether they survived up to 90 days after the index date. In a sensitivity analysis, we will repeat the primary analyses after restricting the cohort to only those who survived at least 90 days after the index date. To explore the effect of our definition of opioid cessation, we will repeat the analysis using a pause in medication supply of 30 days or more instead of 45 days or more, as the definition of cessation of opioid use. Given the uncertainties related to the length of the look-back time window for opioid exposure, we will test our definition of opioidnaive by evaluating exposure with 365 instead of 180 days. Moreover, we will repeat the analysis by changing the definition of persistent opioid use to 61-365 days after the index date.

\section{Missing value analysis}

We will run Little's Missing Completely at Random (MCAR) test to assess whether missing values in the final datasets are MCAR. ${ }^{38}$ If the data are MCAR, multiple imputations with chained equations will be conducted to impute missing values. We will use the 'mice' function in the R software package to perform multiple imputations and the subsequent analysis. We will perform up to five imputations to generate five datasets, and the average of the five values will replace each missing value in each of the five datasets.

\section{Patient and public involvement}

The study was presented at a Māori hui (gathering), Taia Te Hauora, to discuss study with Māori research advisors on study's Māori responsiveness. Our team includes MM (Ngāti Rangiwewehi, Rangitāne ki Manawatū) and the ethics approval process included consultation with a Māori research advisor external to our team. The consultation included information on how best to disseminate study results to Māori.

\section{DISCUSSION}

To our knowledge, this study will result in the most extensive collection of data over the longest period that examines the contribution of surgery or trauma to persistent opioid use and related adverse outcomes explicitly. As NZ has a unique mixture of ethnicities, including an indigenous Māori population, our study will have a particular focus in line with our obligations under the Treaty of Waitangi to assess outcomes related to Māori. ${ }^{16}$
The definition of persistent opioid use has varied in the literature. A recent systematic review found 29 definitions of persistent opioid use. ${ }^{39}$ A 100 -fold difference in the rate of persistent opioid has been reported due to the use of different definitions. ${ }^{39}$ The review supports our interpretation of persistent opioid use. Continuous opioid use was shown to be a more accurate representation of persistent opioid use than single dispensing of opioids within a set period (90-180 days) and a better predictor of subsequent harm. ${ }^{39}$

Our study's main limitation is its reliance on the information collected and coded in administrative datasets. Opioid consumption is extrapolated from pharmacy dispensing data, so medication consumption is not guaranteed. We will not have access to the indication for the continuation of opioid prescribing. Other limitations will include a follow-up period for secondary outcomes of only 365 days. We believe that 365 days would be an adequate time frame for events to occur related to opioid prescribed from the index event date. Although our study extends over a long time period, there have been relatively few changes to prescribing guidelines and opioid availability in NZ during this period. The only significant changes that have occurred were the introduction of oxycodone in 2005 and topical fentanyl patch in $2010 .{ }^{40}$

We aim to capture the effect of introducing these two products to the NZ market and the effect on prescribing of other opioids, for example, morphine. We chose a start date of 1 January 2006 because data for pharmacy dispensing and hospital admissions are more reliable from this date.

We plan to use the M3I over other recognised comorbidity indices such as Charlson or Elixhauser due to its superior prognostic performance in the NZ population. ${ }^{36}$ We will also use ASA for comorbidity as it is readily available on the NMDS, but we will assess which score performs the best. As our study is registry based, we will not have access to each patient's perioperative anaesthetic assessments for the most accurate ASA classification.

\section{ETHICS AND DISSEMINATION}

\section{Data storage, retention and access}

The Research team will not, at any time receive identifying data for any individual in the cohort as the data obtained from the $\mathrm{MOH}$ will be deidentified via encrypted NHIs. Data will be stored in a password-protected folder on the University of Auckland server, managed by the School of Pharmacy's pharmacoepidemiology research group. Data to be stored up to 10 years by the University of Auckland according to the Health Information Privacy Act. ${ }^{41}$ Data governance will be as per the National Ethical Standards for Health and Disability Research 2019. ${ }^{42}$ Only the primary and coinvestigators will be allowed access to data, and data will be stored in a digital deidentified form.

Refer to data management plan in online supplemental material 1 . 


\section{Dissemination}

The findings of this research will be relevant to multiple groups of people, include hospital policy stakeholders, surgeons, anaesthetists, ED physicians and other clinicians who have an interest in acute pain management and subsequent complications. We plan to disseminate our findings at scientific conferences and in high impact peer-reviewed journals that are read by a wide range of users. We plan to have an end of study hui to engage with Māori health users to disseminate study results. As the study uses routinely collected health data, results will be reported per the Reporting of studies Conducted using Observational Routinely collected health Data statement (RECORD) ${ }^{43}$

\section{Author affiliations}

${ }^{1}$ School of Pharmacy, The University of Auckland Faculty of Medical and Health Sciences, Auckland, New Zealand

${ }^{2}$ Pharmacy Department, Auckland District Health Board, Auckland, New Zealand ${ }^{3}$ Department of Anaesthesiology, The University of Auckland Faculty of Medical and Health Sciences, Auckland, New Zealand

${ }^{4}$ Department of Anaesthesia and Perioperative Medicine, Auckland District Health Board, Auckland, New Zealand

${ }^{5}$ Department of Psychological Medicine, University of Otago, Christchurch, New Zealand

${ }^{6}$ Department of Surgery, The University of Auckland Faculty of Medical and Health Sciences, Auckland, New Zealand

\section{Twitter Amy Hai Yan Chan @amyhychan}

Acknowledgements We would like to thank Mr Luke Boyle for the provision of procedure codes and complexity categorisation.

Contributors This study idea was conceived and designed by JG, PJ, AC, DC and AFM. JG, PJ, AC, AFM, MM, KAB, DC, CF and JM provided input to the study design and research questions. JG, KAB and CF developed the statistical plan. JG and $A C$ obtained ethics approval for the study. JG completed the first draft of the manuscript. All authors reviewed and approved the final draft.

Funding This publication was supported by the Auckland Academic Health Alliance (AAHA) grant number 8753 .

Competing interests $\mathrm{JG}$ receives grants from Auckland Academic Health Alliance (AAHA) and scholarship from the University of Auckland. KAB reports grants from A+ charitable trust, New Zealand Pharmacy Education and Research Foundation, outside this submitted work, and U21 Health Sciences Research Development Fund. AC reports consultancy fees from Janssen-Cilag, and from Spoonful of Sugar, a UCL-Business spin-out company, and grants from Innovate UK, A+ charitable trust (Auckland District Health Board), and Maurice and Phyllis Paykel trust, outside this submitted work. DC reports receiving grants from the ADHB Research Trust, the Australian and New Zealand College of Anaesthetists, the Auckland Academic Health Alliance, the Auckland Medical Research Foundation and the Neurological Foundation of New Zealand.

Patient consent for publication Ethics approval includes a waiver of the requirement for patient consent.

Provenance and peer review Peer reviewed for ethical and funding approval prior to submission by the AAHA grant committee.

Supplemental material This content has been supplied by the author(s). It has not been vetted by BMJ Publishing Group Limited (BMJ) and may not have been peer-reviewed. Any opinions or recommendations discussed are solely those of the author(s) and are not endorsed by BMJ. BMJ disclaims all liability and responsibility arising from any reliance placed on the content. Where the content includes any translated material, BMJ does not warrant the accuracy and reliability of the translations (including but not limited to local regulations, clinical guidelines, terminology, drug names and drug dosages), and is not responsible for any error and/or omissions arising from translation and adaptation or otherwise.

Open access This is an open access article distributed in accordance with the Creative Commons Attribution Non Commercial (CC BY-NC 4.0) license, which permits others to distribute, remix, adapt, build upon this work non-commercially, and license their derivative works on different terms, provided the original work is properly cited, appropriate credit is given, any changes made indicated, and the use is non-commercial. See: http://creativecommons.org/licenses/by-nc/4.0/.

\section{ORCID IDs}

Jiayi Gong http://orcid.org/0000-0001-6356-256X

Alan Forbes Merry http://orcid.org/0000-0001-7100-009X

Amy Hai Yan Chan http://orcid.org/0000-0002-1291-3902

\section{REFERENCES}

1 US centres for Disease Control and Prevention. Drug overdose deaths in the United States hit record numbers in 2014: US centres for Disease Control and Prevention; 2014 [cited 2020 1st January]. Available: http://www.cdc.gov/drugoverdose/epidemic/ [Accessed 1 January 2020].

2 Shipton EE, Shipton AJ, Williman JA, et al. Deaths from opioid overdosing: implications of coroners' inquest reports 2008-2012 and annual rise in opioid prescription rates: a population-based cohort study. Pain Ther 2017;6:203-15.

3 Health Quality and Safety Commission New Zealand. Atlas of Healthcare Variation- opioids: HQSC New Zealand; 2017 [cited 2019 27th August]. Available: https://www.hqsc.govt.nz/our-programmes/ health-quality-evaluation/projects/atlas-of-healthcare-variation/ opioids/

4 Schug SA, Lavand'homme P, Barke A, et al. The IASP classification of chronic pain for ICD-11: chronic postsurgical or posttraumatic pain. Pain 2019;160:45-52.

5 Stark N, Kerr S, Stevens J. Prevalence and predictors of persistent post-surgical opioid use: a prospective observational cohort study. Anaesth Intensive Care 2017;45:700-6.

6 Barnett ML, Olenski AR, Jena AB. Opioid-Prescribing patterns of emergency physicians and risk of long-term use. N Engl J Med Overseas Ed 2017;376:663-73.

7 Lopez-Quintero C, Hasin DS, de Los Cobos JP, et al. Probability and predictors of remission from life-time nicotine, alcohol, cannabis or cocaine dependence: results from the National epidemiologic survey on alcohol and related conditions. Addiction 2011;106:657-69.

8 Argyriou E, Um M, Carron C, et al. Age and impulsive behavior in drug addiction: a review of past research and future directions. Pharmacol Biochem Behav 2018;164:106-17.

9 von Oelreich E, Eriksson M, Brattström O, et al. Risk factors and outcomes of chronic opioid use following trauma. Br J Surg 2020;107:413-21.

10 New Zealand (NZ) Ministry of Health. New Zealand health system: Ministry of Health NZ; 2018 [cited 2020 1st May]. Available: https:// www.health.govt.nz/new-zealand-health-system

11 Pharmac. Costs of medicines: Pharmac; 2020 [cited 2020 5th June]. Available: https://www.pharmac.govt.nz/medicines/medicinesinformation/costs-of-medicines/

12 New Zealand (NZ) Ministry of Health. The National Health Index (NHI) number: Ministry of Health NZ; [cited 2019 22nd December]. Available: https://www.health.govt.nz/our-work/health-identity/ national-health-index

13 Jerath A, Austin PC, Wijeysundera DN. Days alive and out of hospital: validation of a patient-centered outcome for perioperative medicine. Anesthesiology 2019;131:84-93.

14 Davis P, Lay-Yee R, Dyall L, et al. Quality of hospital care for Māori patients in New Zealand: retrospective cross-sectional assessment. The Lancet 2006;367:1920-5.

15 Tomlin A, Woods DJ, Lambie A, et al. Ethnic inequality in nonsteroidal anti-inflammatory drug-associated harm in New Zealand: a national population-based cohort study. Pharmacoepidemiol Drug Saf 2020;29:881-9.

16 Treaty of Waitangi [English version] 1840.

17 New Zealand (NZ) Ministry of Health. The National Minimum Dataset (NMDS): Ministry of Health NZ; [cited 2019 19th August]. Available: http://www.health.govt.nz/nz-healthstatistics/national-collectionsand-surveys/collections/national-minimum-dataset-hospital-events

18 The Australian Consortium for Classification Development (ACCD). The Australian Classification of Health Interventions (ACHI) 2019 [cited 2020 20th May].

19 Campbell D, Boyle L, Soakell-Ho M, et al. National risk prediction model for perioperative mortality in non-cardiac surgery. $\mathrm{Br} J$ Surg 2019;106:1549-57.

20 New Zealand (NZ) Ministry of Health. National Non-Admitted Patients Collection (NNPAC): Ministry of Health NZ; [cited 2019 30th December]. Available: https://www.health.govt.nz/nz-health- 
statistics/national-collections-and-surveys/collections/national-nonadmitted-patient-collection

21 (ACC) ACC. Who we are: Accident Compensation Corporation (ACC); [cited 2020 21st July]. Available: https://www.acc.co.nz/about-us/ who-we-are/what-we-do/?smooth-scroll=content-after-navs

22 New Zealand (NZ) Ministry of Health. The Mortality Collection: Ministry of Health NZ; [cited 2019 13th March]. Available: https:// www.health.govt.nz/nz-health-statistics/national-collections-andsurveys/collections/mortality-collection

23 New Zealand (NZ) Ministry of Health. Pharmaceutical Collection dataset: Ministry of Health NZ; [cited 2019 27th August]. Available: https://minhealthnz.shinyapps.io/datapharm/

24 New Zealand (NZ) Ministry of Health. The Primary Health Organisation Enrolment Collection (PHO): Ministry of Health NZ; [cited 2019 30th December]. Available: https://www.health.govt.nz/ nz-health-statistics/national-collections-and-surveys/collections/ primary-health-organisation-enrolment-collection

25 Schneeweiss S, Rassen JA, Brown JS, et al. Graphical Depiction of longitudinal study designs in health care databases. Ann Intern Med 2019;170:398-406

26 Schug SA, Palmer GM, Scott DA, et al. Acute pain management: scientific evidence, fourth edition, 2015. Med J Aust 2016;204:315-7.

27 Dunn KM, Saunders KW, Rutter CM, et al. Opioid prescriptions for chronic pain and overdose: a cohort study. Ann Intern Med 2010;152:85-92.

28 Braden JB, Russo J, Fan M-Y, et al. Emergency department visits among recipients of chronic opioid therapy. Arch Intern Med 2010;170:1425-32.

29 New Zealand Formulary. Opioid analgesics- Switching opioids: New Zealand Formulary; 2020 [cited 2020 27th May]. Available: https:// nzf.org.nz/nzf 70672\#nzf 70708

30 Lalic S, Gisev N, Bell JS, et al. Predictors of persistent prescription opioid analgesic use among people without cancer in Australia. $\mathrm{Br} \mathrm{J}$ Clin Pharmacol 2018;84:1267-78.

31 Brummett CM, Waljee JF, Goesling J, et al. New persistent opioid use after minor and major surgical procedures in US adults. JAMA Surg 2017; 152:e170504.
32 Clarke H, Soneji N, Ko DT, et al. Rates and risk factors for prolonged opioid use after major surgery: population based cohort study. BMJ 2014;348:g1251.

33 Atkinson JSC, Crampton P. NZDep2013 index of deprivation. University of Otago, Dunedin, 2014.

34 Pasternak RL. Preanesthesia evaluation of the surgical patient. ASA Refresher Courses in Anesthesiology 1996;24:205-19.

35 American Society of Anesthesiologists. ASA Physical Status Classification System: American Society of Anesthesiologists; 2019 [cited 2020 1st June]. Available: https://www.asahq.org/standardsand-guidelines/asa-physical-status-classification-system

36 Stanley J, Sarfati D. The new measuring multimorbidity index predicted mortality better than Charlson and Elixhauser indices among the general population. J Clin Epidemiol 2017;92:99-110.

37 Rhon DI, Snodgrass SJ, Cleland JA, et al. Predictors of chronic prescription opioid use after orthopedic surgery: derivation of a clinical prediction rule. Perioper Med 2018;7:25.

38 Roderick JAL. A test of missing completely at random for multivariate data with missing values. J Am Stat Assoc 1988;83:1198-202.

39 Jivraj NK, Raghavji F, Bethell J, et al. Persistent postoperative opioid use: a systematic literature search of definitions and populationbased cohort study. Anesthesiology 2020;132:1528-39.

40 Pharmac. Application tracker: Pharmac; 2020 [cited 2020 16th December]. Available: https://connect.pharmac.govt.nz/apptracker/ $\mathrm{s} /$

41 Privacy Commissioner. Health Information Privacy Code 1994: Privacy Commissioner; 2013 [cited 2020 19th April]. Available: https://www.privacy.org.nz/the-privacy-act-and-codes/codes-ofpractice/health-information-privacy-code-1994/

42 National Ethics Advisory Committee. National Ethical Standards for Health and Disability, and Quality Improvement: Ministry of Health of NZ; 2019 [cited 2020 20th Feb]. Available: https://neac.health.govt. $\mathrm{nz} /$ publications-and-resources/neac-publications/national-ethicalstandards-health-and-disability

43 Benchimol El, Smeeth L, Guttmann A, et al. The reporting of studies conducted using observational Routinely-collected health data (record) statement. PLoS Med 2015;12:e1001885. 